\title{
Thermodynamic properties of metastable Ag-Cu alloys
}

\author{
R. Najafabadi and D. J. Srolovitz \\ Department of Materials Science and Engineering, University of Michigan, Ann Arbor, Michigan 48109 \\ E. Ma and M. Atzmon \\ Department of Nuclear Engineering, University of Michigan, Ann Arbor, Michigan 48109
}

(Received 5 March 1993; accepted for publication 19 May 1993)

The enthalpies of formation of metastable fcc Ag-Cu solid solutions, produced by ball milling of elemental powders, were determined by differential scanning calorimetry. Experimental thermodynamic data for these metastable alloys and for the equilibrium phases are compared with both calculation of phase diagrams (CALPHAD) and atomistic simulation predictions. The atomistic simulations were performed using the free-energy minimization method (FEMM). The FEMM determination of the equilibrium $\mathrm{Ag}-\mathrm{Cu}$ phase diagram and the enthalpy of formation and lattice parameters of the metastable solid solutions are in good agreement with the experimental measurements. CALPHAD calculations made in the same metastable regime, however, significantly overestimate the enthalpy of formation. Thus, the FEMM is a viable alternative approach for the calculation of thermodynamic properties of equilibrium and metastable phases, provided reliable interatomic potentials are available. The FEMM is also capable of determining such properties as the lattice parameter which are not available from CALPHAD calculations.

\section{INTRODUCTION}

Metastable alloys are of great interest due to their novel structures, properties, and applications compared to their equilibrium counterparts. These nonequilibrium phases, either crystalline, nanocrystalline, or amorphous, have received considerable attention since they can now be readily produced through a variety of techniques. ${ }^{1}$ These phases also present challenges to the current understanding of phase transformations in alloy systems. Knowledge of the thermodynamic functions is of primary importance in understanding the formation and stability of these metastable phases.

Determination and calculation of thermodynamic functions for alloys have been the subjects of active research for many years. ${ }^{2-7}$ For equilibrium alloys, the calculations of phase diagrams (CALPHAD) approach ${ }^{2-4}$ is a well-established and widely applied method for calculation of thermodynamic functions and phase diagrams. This method uses analytical expressions to describe the free energy of different phases in an alloy system. In the general formalism, the free energy $G$ of a phase in a binary system is usually expressed as

$G=G_{1} x_{1}+G_{2} x_{2}+k_{B} T\left(x_{1} \ln x_{1}+x_{2} \ln x_{2}\right)+G_{e x}$,

where $G_{1}$ and $G_{2}$ are the free energies (termed lattice stabilities in some publications) for the pure elements, $x_{1}$ and $x_{2}$ the molar fraction of the components, $T$ the temperature, $k_{B}$ the Boltzmann constant, and $G_{\text {ex }}$ the excess free energy of mixing. $G_{1}$ and $G_{2}$ are described by polynomials in $T$, and $G_{\text {ex }}$ by a polynomial in $x$ and $T$. The expressions for $G_{1}, G_{2}$, and $G_{\text {ex }}$ are derived from models, with coefficients ubtained from optimized numerical fits to known thermodynamic data and phase equilibria. ${ }^{2-4}$ In recent years, the use of the CALPHAD method has been ex- tended to the calculation of thermodynamic functions for metastable alloys. ${ }^{8-10}$ However, not surprisingly, CALPHAD extrapolations are prone to significant errors when used for metastable phases, due to input data that are limited to the equilibrium regime, errors introduced during fitting, and invalid extrapolations. ${ }^{9}$ Some modifications, ${ }^{11,12}$ or experimental data for the metastable phases, ${ }^{10}$ have to be incorporated in order to improve the CALPHAD results. There are also other models for calculating thermodynamic functions of metastable alloys. One commonly used method to estimate the enthalpy of formation for binary alloys is the empirical Miedema model. ${ }^{7}$ This model, although qualitatively successful for many systems, does not account for different crystal structures, and often disagrees with experimental data. Therefore, calculations of thermodynamic functions for metastable alloys remain difficult and unreliable, and new methodologies are desirable.

We have recently developed a simulation approach, the free-energy minimization method (FEMM), which is based upon the minimization of an approximate free energy functional with respect to positions of all atoms and the spatial distribution of solutes. In addition to providing thermodynamic functions such as the free energy and entropy of equilibrium alloys, the FEMM also allows determination of thermodynamic functions of metastable alloys and defects. A detailed description of this simulation procedure has been given in Ref. 13 and will be briefly described in Sec. II. In this paper, we apply FEMM simulations to calculate thermodynamic functions for metastable phases in $\mathrm{Ag}-\mathrm{Cu}$ system. A systematic comparison with experimental enthalpy data and CALPHAD calculations is performed.

The present paper focuses on metastable solid solutions 
in the Ag-Cu binary system. The eutectic $\mathrm{Ag}-\mathrm{Cu}$ system was the first system in which Duwez et al. successfully produced continuous, metastable fcc solid solutions using the rapid quenching technique. ${ }^{14}$ Over the years, a number of other techniques have also been applied to form these solutions. ${ }^{15,16}$ In this work, we chose to use the technique of ball milling of elemental powders, which is a lowtemperature mechanical alloying process. ${ }^{17}$ Previous experimental characterization of the thermodynamic functions of $\mathrm{Ag}-\mathrm{Cu}$ solutions has been sketchy. ${ }^{18}$ The easy formation of relatively large quantities of these solid solutions by ball milling allowed us to determine the enthalpy of formation $(\Delta H)$ from differential scanning calorimetry measurements.

CALPHAD fitting has been performed to reproduce the equilibrium phase diagram for this system. ${ }^{5}$ However, the CALPHAD expressions, obtained by extrapolation from narrow equilibrium solubility ranges, are unlikely to properly describe metastable concentrated solid solutions. The embedded atom method (EAM) interatomic potentials ${ }^{19}$ used in the FEMM simulations have been well established for this system. In Sec. IV, it is shown that the phase diagram determined experimentally and that determined using the FEMM and EAM potentials agree well with each other. FEMM calculations are shown to provide good agreement with our experimental measurements of the lattice parameters and enthalpies of formation of metastable Ag-Cu solid solutions. In contrast, the CALPHAD method significantly overestimates the enthalpies of formation of these alloys.

\section{FREE-ENERGY MINIMIZATION METHOD}

The Gibbs free energy of an alloy system may be written as

$$
G=E_{\text {bond }}+E_{\text {vib }}+E_{\text {conf }} .
$$

The three components of the free energy are the bonding energy ( $E_{\text {bond }}$ ), the contribution of atomic vibrations $\left(E_{\mathrm{vib}}\right)$, and the contributions due to the configurational entropy term ( $\left.E_{\text {conf }}\right)$.

The bonding energy within the EAM framework ${ }^{19}$ is given as

$$
E_{\text {bond }}\left(r_{1}, \ldots, r_{N}\right)=\sum_{i=1}^{N}\left\{F_{i}\left[\rho\left(r_{i}\right)\right]+\frac{1}{2} \sum_{j=1}^{N} \phi\left(r_{i j}\right)\right\},
$$

where $r_{i}$ is the position of the $i$ th atom, $N$ is the total number of atoms in the system, and $r_{i j}$ is the separation between atoms $i$ and $j$. The $\phi\left(r_{i j}\right)$ is a repulsive pair potential representing the interaction between the ion cores of atoms $i$ and $j$. The embedding energy function $F_{i}(\rho)$ is the energy required to insert atom $i$ into a medium with a uniform electron charge density $\rho$. The electron density at site $i$ is assumed to be a superposition of the electron densities due to the neighboring atoms

$$
\rho\left(r_{i}\right)=\sum_{j=1}^{N} \rho_{j}\left(r_{i j}\right)
$$

where $\rho_{j}\left(r_{i j}\right)$ is the electron density of atom $j$ at the distance $r_{i j}$ from its nucleus.

The vibrational contribution to the free energy is determined within the framework of the local harmonic (LH) model. ${ }^{20}$ This model has been applied with considerable success to perfect and defected single-component solids. ${ }^{21}$ In this model, each atom is assumed to vibrate within a potential well which may be characterized by three independent frequencies. These frequencies are determined in terms of the local dynamical matrix of each atom $D_{i \alpha \beta}=\left(\partial^{2} E_{\text {bond }} / \partial r_{i \alpha} \partial r_{i \beta}\right)$, where $r_{i \alpha}$ is the coordinate of the $i$ th atom in the $\alpha$ direction. In this picture, the direct coupling between the vibrational modes of different atoms is ignored. The classical vibrational contribution to the free energy within this model is

$$
E_{\mathrm{vib}}=k_{B} T \sum_{i=1}^{N} \sum_{\beta=1}^{3} \ln \frac{\hbar \omega_{i \beta}}{k_{B} T},
$$

where $\hbar$ is Planck's constant and $\omega_{i 1}, \omega_{i 2}$, and $\omega_{i 3}$ are the three vibrational frequencies of atom $i$.

The configurational entropy contribution $E_{\text {conf }}$ to the free energy of a binary alloy, ignoring the short-range order, is written using the ideal entropy of mixing (BraggWilliams approximation) as

$E_{\mathrm{conf}}=-k_{B} T \sum_{i=1}^{N}\left\{x_{a}(i) \ln \left[x_{a}(i)\right]+x_{b}(i) \ln \left[x_{b}(i)\right]\right\}$,

where $x_{a}(i)$ is the probability that atomic site $i$ is occupied by an atom of type $a$, and correspondingly, $x_{b}(i)=1-x_{a}(i)$ is the probability that the same site is occupied by an atom of type $b$. For a more detailed description of the free-energy minimization method and its applications one may refer to Ref. 13.

In the present simulations, the Gibbs free energy is minimized with respect to the lattice parameters describing the unit cell. Although the initial unit cell has an fcc structure, during the course of the minimization, the unit cell may deform to any other structure. In order to simulate a metastable phase, we simply impose an appropriate symmetry on the site concentrations. Therefore, in order to simulate a uniform solid solution, we fix the concentrations on all atomic sites to be the same and then minimize the free energy (with respect to the atomic positions) for a series of bulk compositions at several temperatures.

\section{EXPERIMENTAL METHOD}

A mechanical alloying technique, high-energy ball milling, was used to form metastable Ag-Cu solid solutions. Eight grams of powders of commercially available $\mathrm{Ag}$ and $\mathrm{Cu}$ were blended into a mixture with overall composition $\mathrm{Ag}_{100-y} \mathrm{Cu}_{y}$ for nine different values of $y$ between 0 and 100 . These powders were milled with stainless-steel balls at a ball-to-powder weight ratio of $4: 1$ in a stainlesssteel vial using a SPEX $8000 \mathrm{miller} / \mathrm{mixer}$, for a duration of $40 \mathrm{~h}$. This duration was chosen after test runs for selected compositions-no further changes were observed upon milling longer than $40 \mathrm{~h}$. A fan was used to keep the milling vial temperature close to $35^{\circ} \mathrm{C}$. X-ray diffraction 


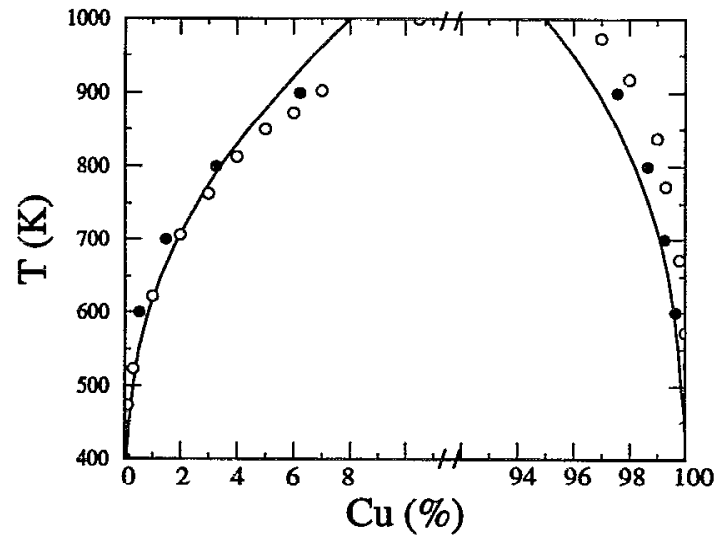

FIG. 1. The solid portion of the Ag-Cu phase diagram. The solid line is determined using the free-energy minimization method and EAM interaction potentials. The open and closed circles represent CALPHAD calculation results and experimental data, respectively.

was employed for phase identification. Heat evolution during phase transformation to equilibrium in milled powders was monitored using a calibrated differential scanning calorimeter (Perkin-Elmer DSC-7) and correlated with the $\mathrm{x}$-ray diffraction results. The baseline, determined by repeating each run without disturbing the sample, was subtracted from each run. Energy dispersive x-ray spectroscopy was conducted in a scanning electron microscope for analysis of the composition of the milled powders. Using energy-dispersive $\mathrm{x}$-ray analysis, neither composition heterogeneity nor contamination from the milling tools were detected.

\section{RESULTS}

We first performed a series of FEMM simulations for equilibrium $\mathrm{Ag}-\mathrm{Cu}$ alloys in the solid state. Figure 1 shows the solid regions of the $\mathrm{Ag}-\mathrm{Cu}$ phase diagram determined from experiment, ${ }^{6}$ from FEMM simulations, and from CALPHAD data. ${ }^{5}$ Good agreement between FEMM and the experimentally determined phase boundaries is observed. This agreement is significant in a number of respects: (i) it enhances our confidence in the EAM potentials we employ to describe the interatomic interaction in the Ag-Cu system; (ii) it further demonstrates the validity of the FEMM simulation procedure, which has successfully been applied in other applications; ${ }^{22-25}$ it suggests (iii) that the FEMM with EAM potentials is a reasonable alternative to existing methods in the calculation of equilibrium phase diagrams; and (iv) that FEMM/EAM may be reliable in the metastable regime.

We calculated the enthalpy of formation, $\Delta H$, and free energy of formation, $\Delta G$, for extended Ag-Cu solid solutions using FEMM simulations. The pure elements are used as the reference state throughout this paper. A plot of $\Delta H$ vs $\mathrm{Cu}$ concentration $x$ is shown as the solid curve in Fig. 2. For comparison, the dashed curve is calculated using previously published CALPHAD data. ${ }^{5}$ Also included are data points for equilibrium solid solutions assessed by Hultgren, ${ }^{6}$ based on dissolution calorimetry data (open cir-

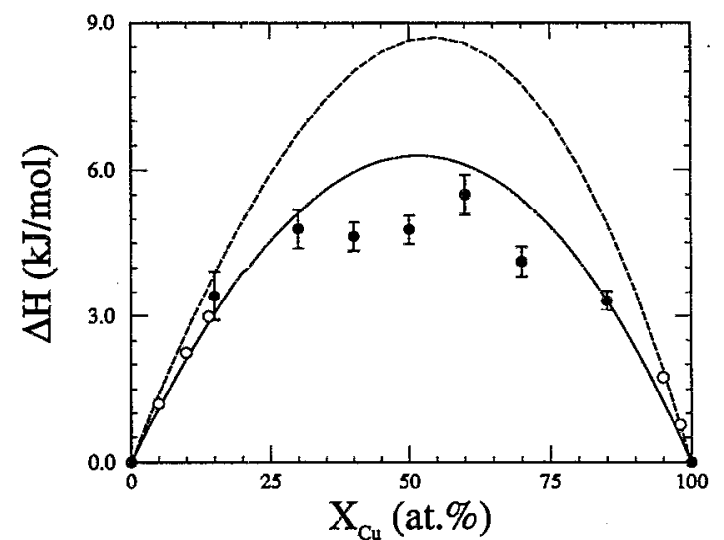

FIG. 2. Enthalpy of formation of the Ag-Cu alloy at $700 \mathrm{~K}$ as a function of $\mathrm{Cu}$ concentration. The solid and dashed lines are the results of FEMM simulation and CALPHAD calculations, respectively. The open and filled circles represent the experimental data for the equilibrium phase (Ref. 6) and present measurements for the metastable phase, respectively.

cles), and our present experimental data for metastable $\mathrm{Ag}-\mathrm{Cu}$ solid solutions (filled circles), as detailed below.

We formed continuous fcc solid solutions by highenergy ball milling elemental $\mathrm{Ag}$ and $\mathrm{Cu}$ powders. Figure 3 (a) shows the $\mathrm{x}$-ray diffraction pattern for a $40 \mathrm{~h}$ milled $\mathrm{Ag}_{60} \mathrm{Cu}_{40}$ powder mixture. A single set of fcc Bragg peaks is present with positions between those for pure $\mathrm{Ag}$ and pure $\mathrm{Cu}$. After heating in a differential scanning calorimeter (DSC) at $20 \mathrm{~K} / \mathrm{min}$ to $900 \mathrm{~K}$, peaks for both fcc $\mathrm{Ag}$ and $\mathrm{Cu}$ were observed [Fig. 3(b)] at nearly the same angles as for the pure elements. These results demonstrate that ball milling indeed produced a single fcc solid solution

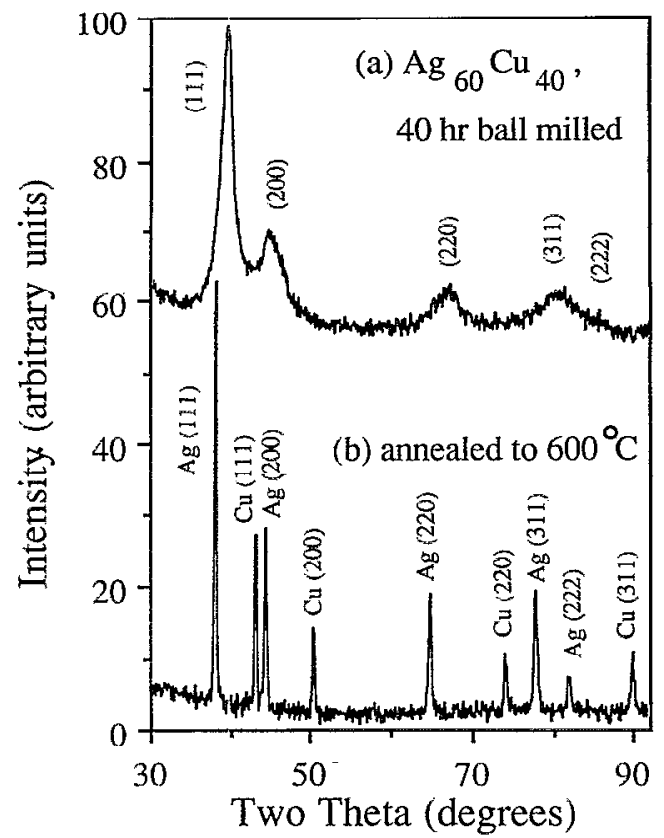

FIG. 3. X-ray diffraction patterns for (a) $40 \mathrm{~h}$ milled $\mathrm{Ag}_{60} \mathrm{Cu}_{40}$ powder, (b) the same powder as in (a), after heating to $900 \mathrm{~K}$ at $20 \mathrm{~K} / \mathrm{min}$. 


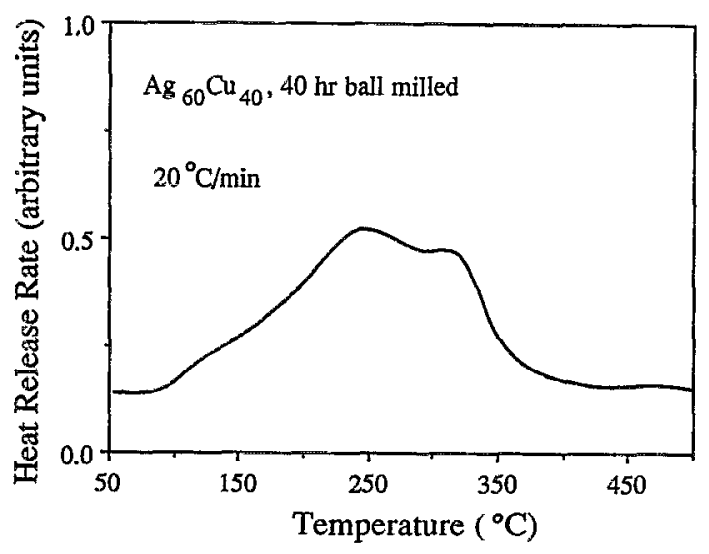

FIG. 4. DSC trace for $40 \mathrm{~h}$ milled $\mathrm{Ag}_{60} \mathrm{Cu}_{40}$ powder, measured at a heating rate of $20 \mathrm{~K} / \mathrm{min}$.

phase. The fcc solutions obtained by ball milling have lattice parameters similar to those formed by rapid quenching ${ }^{26}$ (see discussion below). DSC experiments revealed that the transformation of the metastable solution back to equilibrium terminal solid solutions was complete at approximately $700 \mathrm{~K}$. According to Fig. 1, the equilibrium terminal solutions are $\mathrm{Ag}(2$ at. $\% \mathrm{Cu})$ and $\mathrm{Cu}(1$ at. $\% \mathrm{Ag})$ at $700 \mathrm{~K}$. The heat released during this transformation process is recorded in Fig. 4. Integration of the total area under this heat release curve gives the enthalpy of equilibration. To obtain the enthalpy of formation, $\Delta H$, two additional contributions were taken into account. (i) The enthalpy of formation of the terminal solutions (in equilibrium at $700 \mathrm{~K}$ ) has been added. This term is determined based on data tabulated in Ref. 6. (ii) An estimate of heat release due to recovery and grain growth in the cold-worked material has been subtracted. For alloys, this latter contribution has been estimated using a linear interpolation between the stored enthalpies measured for pure $\mathrm{Ag}(0.45 \mathrm{~kJ} / \mathrm{mol})$ and pure $\mathrm{Cu}(1.08 \mathrm{~kJ} / \mathrm{mol})$ after milling for $40 \mathrm{~h}$. Errors involved in this step are discussed in Sec. V.

The $\Delta H$ values obtained from the corrected enthalpy of equilibration are shown as filled circles in Fig. 2. It can be seen from Fig. 2 that the agreement between the FEMM results and experimental data is good in the equilibrium regime, and is also satisfactory in the metastable regime. In contrast, the CALPHAD results (dashed curve) significantly overestimate $\Delta H$ for metastable solutions, while providing a reasonable fit to the $\Delta H$ data for equilibrium solutions. Figure 2 indicates that $\Delta H$ for the fcc solid solution is positive. According to both FEMM simulations and CALPHAD calculations at different temperatures, $\Delta H$ is nearly independent of temperature up to the eutectic temperature.

The free energy of formation, $\Delta G$, determines the thermodynamic stabilities of the phases. Calculations of $\Delta G$ for the fcc Ag-Cu solution have been performed using FEMM and CALPHAD, and the curves for a temperature of 400 $\mathrm{K}$ are displayed in Fig. 5 with solid and dashed lines, respectively. The $\Delta G$ curve for the liquid phase, calculated

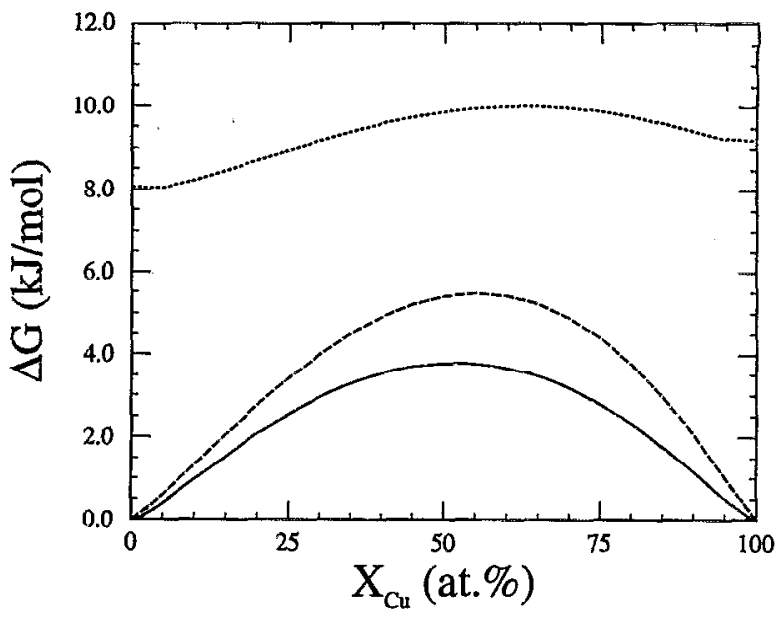

FIG. 5. Free energy of formation of the $\mathrm{Ag}-\mathrm{Cu}$ alloy at $400 \mathrm{~K}$ as a function of $\mathrm{Cu}$ concentration. The solid line is determined using FEMM with EAM interaction potentials. The dashed and dotted lines are the results of CALPHAD calculations for solid and undercooled liquid phase, respectively.

using direct extrapolation of the CALPHAD $\Delta G$ expression to the same temperature, is also included in Fig. 5 (dotted line). These results are discussed in the following section.

FEMM simulations also yield the lattice parameters of the metastable fcc phase. The deviation of the lattice parameter from Vcgard's law versus $\mathrm{Cu}$ concentration is shown in Fig. 6 (solid curve), together with data obtained from our $\mathrm{x}$-ray analysis (filled circles) and from the literature for fcc solutions formed by rapid quenching (open circles) ${ }^{26}$ Due to the broadness of the Bragg peaks for our ball-milled samples the uncertainty in our data is significant. The data shown in Fig. 6 were obtained from the Bragg peaks present in the diffraction pattern which were

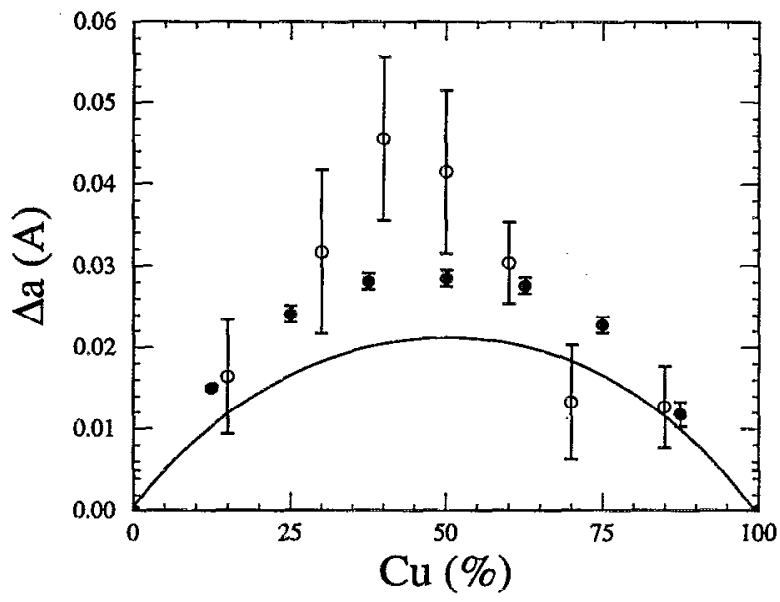

FIG. 6. The deviation of the lattice parameter from the Vegard's law, $\Delta a$ vs $\mathrm{Cu}$ concentration. The solid line is determined using the free-encrgy minimization method and EAM interaction potentials. The open and filled circles represent data obtained from present $x$-ray analysis and from lattice parameter measurement in rapidly quenched samples (Ref. 26), respectively. 
sufficiently intense and did not overlap extensively with other peaks. The lattice parameter data from Ref. 26 contain smaller error because they were obtained from rapidly quenched samples, for which the defect density. was smaller than that of ball-milled alloys. The small, positive deviation from Vegard's law seen in Fig. 6 is in qualitative agreement with the small positive enthalpy of mixing of the system (Fig. 2). Again, good quantitative agreement between the FEMM/EAM simulation and experimental data is obtained. This provides additional support to the reliability of the simulated thermodynamic functions presented above.

\section{DISCUSSION}

We observe the thermodynamic functions and phase equilibria for the Ag-Cu system to be successfully determined by the FEMM/EAM simulations. First, sufficiently far below the eutectic temperature $(1053 \mathrm{~K})$, the equilibrium phase diagram determined by the FEMM simulations and the CALPHAD phase diagram agree with the experimental equilibrium phase diagram to a similar degree. Second, in the metastable regime, the FEMM/EAM has clear advantages over CALPHAD extrapolations. It not only matches experimental $\Delta H$ data significantly better, but also supplies lattice parameter data that are not available from CALPHAD. FEMM hence provides a new approach for calculating thermodynamic functions for metastable phases, which does not suffer most of the difficulties inherent to the CALPHAD method. We note two potential sources of error in the FEMM method: (1) errors in the interatomic potentials; (2) approximations made in deriving the free energy functional employed in the FEMM procedure may introduce some error, especially near the critical points.

We now examine possible sources of error in the DSC measurement of $\Delta H$. First, the DSC signal (e.g., that shown in Fig. 4) is obtained over a wide range of temperature/time. The integration over such wide ranges may involve significant error due to an unavoidable drift of the DSC baseline. The errors bars shown in Fig. 2 are, hence, relatively large. In addition, the DSC signal includes the heat release due to recovery and grain growth in the cold-worked powders. To obtain the $\Delta H$ data shown in Fig. 2, as a first approximation, we have subtracted an amount of heat release equivalent to the weighted average of that observed for pure $\mathrm{Ag}$ or $\mathrm{Cu}$ after the same milling duration (Sec. IV A). However, this approximation may not be sufficient since the alloy may have larger strains and smaller grain sizes than the elemental phases. Thus the subtracted enthalpy may not have fully accounted for the stored energy due to cold work in these alloy powders.

An additional source of error may be the presence of compositional fluctuations in the milled powders due to inefficient mixing, since $\Delta H>0$ provides a driving force for demixing. Based on our diffraction data, we can exclude the presence of significant amounts of unmixed elemental domains. However, we cannot exclude the possibility that small-amplitude fluctuations exist. The effect of such fluctuations on the width of diffraction peaks would be masked by that of structural defects. We notice that the lattice parameters we measured for some alloys in the central composition region are higher than those determined for rapidly quenched samples. ${ }^{26}$ This may be an indication of compositional heterogeneity in these milled samples, since the Ag-rich domains, whose lattice parameter is larger than that of the Cu-rich domains, are weighted more strongly due to their higher scattering factors. Because of the curvature of $\Delta H$ versus composition curve, compositional fluctuations may also cause an underestimate of $\Delta H$. In fact, $\Delta H$ measured at these compositions (Fig. 2) is lower than either the CALPHAD or FEMM results. Considering the magnitude of the curvature of $\Delta H$ versus composition curve, however, we believe that the effect of composition inhomogeneity on the measured $\Delta H$ is unlikely to be very significant.

The magnitude of the heat release due to recovery and grain growth can be estimated as follows. The maximum rms strain observed for similar binary solid solutions was reported to be less than $2 \% .{ }^{27}$ The strain energy contribution to the heat release $u$ can be estimated using $u=(Y / 2)\left\langle e^{2}\right\rangle,{ }^{28}$ where $Y$ is the Young's modulus and $\left\langle e^{2}\right\rangle^{1 / 2}$ is the rms strain. For a value of $Y=130 \times 10^{9} \mathrm{~N} / \mathrm{m}^{2}$ (e.g., for $\mathrm{Cu}){ }^{29}$ the relief of an rms strain of $2 \%$ would give about $0.18 \mathrm{~kJ} / \mathrm{mol}$. Using a typical grain boundary energy of $0.5 \mathrm{~J} / \mathrm{m}^{2}$, the heat release due to grain growth, from 12 to about $40 \mathrm{~nm}$ (as estimated from our $\mathrm{x}$-ray diffraction data), is $\sim 0.75 \mathrm{~kJ} / \mathrm{mol}$. These two terms amount to less than $1 \mathrm{~kJ} / \mathrm{mol}$, which is similar to the subtracted value based on interpolation from the pure elements $(0.4-1.1 \mathrm{~kJ} / \mathrm{mol})$. Although this is a crude estimate, we believe the $\Delta H$ data presented in Fig. 2 are unlikely to be significant overestimates, especially when there may be a compensating effect of compositional inhomogeneity, as described above. We note that an accurate measurement of thermodynamic quantities is difficult for metastable alloys, which, being in a nonequilibrium state, necessarily requires extreme conditions for formation. The resulting alloy usually has small grain sizes, large strains and defect densities. In a DSC measurement, the heat released due to these factors may not always be resolvable from that solely from the phase transformation. ${ }^{30}$

We note that various techniques, including liquid quenching, ${ }^{14}$ vapor deposition, ${ }^{15}$ mechanical alloying, ${ }^{18}$ and ion mixing, ${ }^{16}$ have failed to form an amorphous phase in the Ag-Cu system. A comparison of $\Delta G$ curves at low temperatures $(400 \mathrm{~K})$ for the solid solution and amorphous phase will be useful to understand this observation. The FEMM simulation method requires knowledge of the atomic positions, and thus cannot be applied to liquids. Therefore, we rely on CALPHAD calculations for this discussion. Direct extrapolation of the CALPHAD $\Delta G$ expression for liquid to $400 \mathrm{~K}$ (the upper dotted curve in Fig. 5) will not correctly represent the amorphous phase, since such an extrapolation deep into the undercooled regime necessarily involves large error. It is known that a liquid loses entropy upon continuous undercooling due to the increasing degree of order. This effect gives rise to an excess specific heat in the liquid, $\Delta C_{p}{ }^{31}$ It has been suggested 
that, with undercooling, the specific heat of the liquid eventually becomes very similar to that of the crystallized phases $^{32}$ at a temperature $T_{s}$, defined as the ideal glass transition temperature. Therefore, the entropy loss after undercooling to $T<T_{g}, \Delta S_{1}$, is approximately equal to $\Delta S_{m}$, where $T_{g}\left(\approx T_{s}\right)$ is the experimental glass transition temperature, and $\Delta S_{m}$ the entropy of fusion. For typical glass formers, $T_{m} \approx 2-3 T_{g}$, where $T_{m}$ is the melting temperature. For undercooling to $T<T_{g}$, the accompanying enthalpy loss $\Delta H_{1}$ has been estimated ${ }^{11}$ to be about $(2 / 3) \Delta H_{m}$ for several simple functional forms of $\Delta C_{p}$, where $\Delta H_{m}=\Delta S_{m} T_{m}$ is the enthalpy of fusion. Thus, the corresponding free-energy change, $\Delta G_{1}=\Delta H_{1}-T \Delta S_{1}$, is estimated to be $1 / 3 \Delta H_{m}$, which is on the order of $4 \mathrm{~kJ} /$ mol for $\mathrm{Ag}$-Cu alloys (the enthalpy of fusion of $\mathrm{Ag}$ and $\mathrm{Cu}$ are 11 and $13 \mathrm{~kJ} / \mathrm{mol}$, respectively). This estimated correction term would lower the CALPHAD liquid curve by $4 \mathrm{~kJ} / \mathrm{mol}$, but the resulting curve would still be above that of the fcc phase. Although this estimate is crude, it provides an indication that the amorphous phase (undercooled liquid) is thermodynamically unstable with respect to the fcc phase for the entire composition range. The fcc phase will form as long as the simple fcc structure is not suppressed by kinetic constraints. This is in agreement with experimental observations. ${ }^{14-16,18}$

\section{CONCLUSIONS}

Atomistic simulations based upon the free-energy minimization method (FEMM) and embedded atom method (EAM) interatomic potentials have been conducted to determine the enthalpy of formation $(\Delta H)$ and free energy of formation $(\Delta G)$ of metastable and equilibrium $\mathrm{Ag}-\mathrm{Cu}$ alloys. The FEMM determination of the Ag-Cu equilibrium phase diagram is in good agreement with experiment. Continuous fcc $\mathrm{Ag}-\mathrm{Cu}$ solid solutions have been formed by mechanical alloying of elemental powders. The enthalpy of formation has been measured using differential scanning calorimetry (DSC). Over the entire metastable regime, the enthalpy of formation and lattice parameters determined by the FEMM/EAM simulations are in good agreement with our experimental measurements. CALPHAD calculations performed for the metastable regime, however, significantly overestimate the enthalpy of formation. Thus, the FEMM is a viable alternative approach for the calculation of thermodynamic properties of equilibrium and metastable phases, provided reliable interatomic potentials are available. The FEMM also provides data on such properties as the lattice parameter that are not available from CALPHAD calculations.

\section{ACKNOWLEDGMENTS}

E.M. and M.A. gratefully acknowledge support of the National Science Foundation, Grant No. DMR-9200132. R.N. and D.J.S. gratefully acknowledge the support of the Division of Materials Science of the Office of Basic Energy Sciences of the U.S. Department of Energy, Grant No. FG02-88ER45367.

${ }^{1}$ W. L. Johnson, Prog. Mater. Sci. 30, 81. (1986).

${ }^{2}$ L. Kaufman and L. Bernstein, Computer Calculations of Phase Diagrams (Academic, New York, 1970).

${ }^{3}$ J. L. Murray, Bull. Alloy Phase Diagr. 4, 81 (1983).

${ }^{4}$ N. Saunders, CALPHAD 9, 297 (1985).

${ }^{5}$ J. L. Murray, Met. Trans. A 15, 261 (1984).

${ }^{6}$ R. Hultgren, P. D. Desai, D. T. Hawkins, M. Gleiser, and K. K. Kelley, Selected Values of the Thermodynamic Properties of Binary Alloys (ASM, Metals Park, OH, 1973), p. 44.

${ }^{7}$ A. R. Miedema, Philips Tech. Rev. 36, 217 (1976).

${ }^{8}$ N. Saunders and A. P. Miodownik, J. Mater. Res. 1, 38 (1986).

${ }^{9}$ R. B. Schwarz, P. Nash, and D. Turnbull, J. Mater. Res. 2, 456 (1987).

${ }^{30}$ R. Bormann and K. Söltzer, Phys. Status Solidi (A) 131, 691 (1992).

${ }^{11}$ E. Ma and M. Atzmon, J. Alloys Compounds 194, 235 (1993).

${ }^{12}$ E. Ma, M. Atzmon, and F. Pinkerton, J. Appl. Phys. 74, 955 (1993).

${ }^{13}$ R. Najafabadi, H. Y. Wang, D. J. Srolovitz, and R. LeSar, Acta Metall. Mater. 39, 3071 (1991).

${ }^{14}$ P. Duwez, R. H. Willens, and W. Klement, J. Appl. Phys. 31, 1136 (1960).

${ }^{15}$ N. Saunders and A. P. Miodownik, J. Mater. Sci. 22, 629 (1987).

${ }^{16}$ B. Y. Tsaur and J. W. Mayer, Appl. Phys. Lett. 36, 823 (1980).

${ }^{17}$ C. C. Koch, Ann. Rev. Mater. Sci. 19, 121 (1988).

${ }^{18}$ K. Uenishi, K. F. Kobayashi, K. Y. Ishihara, and P. H. Shingu, Mater. Sci. Eng. A 134, 1342 (1991).

${ }^{19}$ S. M. Foiles, M. I. Baskes, and M. S. Daw, Phys. Rev. B 33, 7983 (1986).

${ }^{20}$ R. LeSar, R. Najafabadi, and D. J. Srolovitz, Phys. Rev. Lett. 63, 624 (1989).

${ }^{21}$ R. Najafabadi, D. J. Srolovitz, and R. LeSar, J. Mater. Res. 5, 2663 (1990); 6, 999 (1991).

${ }^{22}$ H. Y. Wang, R. Najafabadi, D. J. Srolovitz, and R. LeSar, Philos. Mag. A 65, 625 (1992).

${ }^{23}$ H. Y. Wang, R. Najafabadi, D. J. Srolovitz, and R. LeSar, Phys. Rev. B 45, 12028 (1992).

${ }^{24}$ R. Najafabadi, H. Y. Wang, D. J. Srolovitz, and R. LeSar, Scr. Metall. Mater. 25, 2497 (1991).

${ }^{25}$ H. Y. Wang, R. Najafabadi, D. J. Srolovitz, and R. LeSar, Met. Trans. 23A, 3105 (1992).

${ }^{26}$ R. K. Linde, J. Appl. Phys. 37, 934 (1966).

${ }^{27}$ J. Eckert and W. L. Johnson (private communication).

${ }^{28}$ M. Atzmon, K. M. Unruh, and W. L. Johnson, J. Appl. Phys. 58, 3865 (1985).

${ }^{29}$ J. Friedel, Dislocations (Pergamon, Oxford, 1964), p. 448.

${ }^{30}$ E. Ma and M. Atzmon, Phys. Rev. Lett. 67, 1126 (1991)

${ }^{31}$ H. S. Chen and D. Turnbull, J. Chem. Phys. 48, 2560 (1968).

${ }^{32}$ M. T. Clavaguera-Mora and N. Clavaguera, J. Mater. Res. 4, 906 (1989). 